\title{
The Debate in Cuba's Scientific Community on Sudden Cardiac Death
}

\author{
Ernesto Vilches MD MS, Luis A. Ochoa MD PhD, Lianne Ramos MD
}

\section{ABSTRACT}

Sudden cardiac death poses a challenge to modern medicine because of its high incidence, the unexpected and dramatic nature of the event, and years of potential life lost. What's more, despite modest decreases in global mortality attributed to cardiovascular diseases, incidence of sudden cardiac death has not declined. Cuba, like most of the Americas, suffers from knowledge gaps that hamper adequate strategies to address sudden cardiac death as a population health problem. We suggest that a generally accepted operational defini-

\section{INTRODUCTION}

Sudden cardiac death (SCD) is a major global public health problem accounting for an estimated $10 \%-30 \%$ of all deaths. [1] Four to five million SCDs are estimated annually worldwide, with 180,000 to 250,000 in the USA alone, accounting for $50 \%$ of the country's ischemic heart disease mortality and the first manifestation of heart disease in $19 \%-26 \%$ of cases.[2] The Vizcaya region of Spain has a reported annual SCD incidence of 13.2 per 100,000 population.[3] Determining the full extent of the problem in the Americas, especially in Cuba, is made difficult by the lack of official SCD registries.[4]

Globally, fewer than $15 \%$ of patients reportedly survive an episode of sudden cardiac arrest, partly because most cases (two thirds) occur outside hospitals. In this context, other influential factors include absence of eyewitnesses or their lack of training in basic cardiopulmonary resuscitation, lack of portable defibrillators in high-traffic public areas, and an erratic chain of survival.[3,5]

There are no recent studies of SCD diagnosis in relation to time of death, so Kuller's work continues to be an essential referent. He found that if sudden death (SD) is defined as death within 2 hours of symptom onset, $12 \%$ of all deaths were classified as sudden, $88 \%$ of these of cardiac origin. If the time frame was extended to $<24$ hours, then $32 \%$ of deaths were considered sudden and only $75 \%$ were due to cardiac causes.[6]

International examples have shown that actions to increase survival must be taken in the first ten minutes following onset of deteriorated cardiovascular function until first contact with specialized care, since probability of survival decreases proportionately with time elapsed following the acute event. Survival probability decreases $10 \%$ for each minute of delay, so that by 12 minutes after acute cardiovascular collapse, the likelihood of surviving is virtually nil.[7] Hospital survival can be as high as $74 \%$ in cases where defibrillation was performed in the first three minutes. [8] In fact, availability of automated external defibrillators linked to public-access defibrillation programs may be the greatest advance in treatment of these patients.[4]

García's study on the impact of Cuba's Integrated Medical Emergency System (SIUM) on mortality from acute myocardial tion of sudden cardiac death be agreed upon, and a national registry developed that recognizes this cause of death on death certificates. These two actions will enable Cuba's public health authorities to assess the extent of the problem and to design intervention strategies for the population with intermediate and lower cardiovascular risk, the group in which most cases occur.

KEYWORDS Sudden cardiac death, cardiovascular disease, sudden death, sudden cardiac arrest, risk reduction, prevention and control, Cuba

infarction found that only $58.3 \%$ of 658 patients reached hospital emergency services through SIUM and that of these, only $41.3 \%$ received thrombolytic reperfusion therapy.[9] The same study noted that SIUM ambulances transferred only $8 \%$ of patients within the first hour of symptom onset.

A necessary starting point for assessing the magnitude of SCD is to reach scientific consensus on an acceptable operational definition. Best scientific practices and clinical competence are also needed, leading to correct interpretation of the term sudden death on the death certificate.[10]

In Cuba, there is statistical underreporting of SCD (Table 1), preventing assessment of the true magnitude of the problem. Despite SCD's inclusion in ICD-10 (code 146.1),[11] most cases are not reported (in 2012 , only $0.04 \%$ of all natural deaths were reported as SCD, much lower than the most conservative estimate cited above, $12 \%)$.[12] There is a misconception that the term sudden cardiac death should be a diagnosis of exclusion, only reportable as cause of death when the direct cause is unknown.[13] This is contradictory since, whether or not cause of death is identified when a sudden death occurs, the diagnostic definition remains the same: an unexpected, natural death occurring in a short period of time.

The Interamerican Society of Cardiology established the Alliance Against Sudden Cardiac Death to convince health authorities in the Americas to consider SCD a public health problem, advocating for its official recognition as a direct cause of death.[14] This would facilitate recording and recovery of official statistics on the epidemiology of SCD in every country in the hemisphere. In 2013, Cuba's Sudden Death Research Group (GIMUS) was accepted as an active member of the Alliance to cooperate internationally in tackling SCD, in recognition of GIMUS's 20-year track record of research fully aligned with this initiative.

The purpose of this paper is to describe and analyze the main SCD issues under discussion in various biomedical specialties (internal medicine, cardiology, emergency medicine, intensive care, pathology, forensic medicine and epidemiology), and to offer recommendations for the Cuban context, in order to better understand and address the magnitude of the SCD problem in our country. 
Table 1: Comparative analysis of reported SCDs and estimates by GIMUS

\begin{tabular}{|l|r|r|r|r|}
\hline & \multirow{2}{*}{$\begin{array}{c}\text { Heart } \\
\text { disease } \\
\text { deaths }\end{array}$} & Estimated & \multicolumn{3}{|c|}{ SCDs } \\
\cline { 4 - 5 } & & & & \multicolumn{2}{c|}{$\begin{array}{c}\text { \% } \\
\text { Reported }\end{array}$} & $\begin{array}{c}\text { (reported } \\
\text { (estimated) }\end{array}$ \\
\hline 2001 & 20,727 & 10,363 & 131 & 1.3 \\
\hline 2002 & 19,078 & 9539 & 118 & 1.2 \\
\hline 2003 & 20,390 & 10,195 & 143 & 1.4 \\
\hline 2004 & 21,056 & 10,528 & 144 & 1.4 \\
\hline 2005 & 22,223 & 11,111 & 140 & 1.3 \\
\hline 2006 & 21,221 & 10,610 & 126 & 1.2 \\
\hline 2007 & 21,048 & 10,524 & 134 & 1.3 \\
\hline 2008 & 22,430 & 11,215 & 132 & 1.2 \\
\hline 2009 & 22,340 & 11,170 & 134 & 1.2 \\
\hline 2010 & 23,904 & 11,952 & 105 & 0.9 \\
\hline 2011 & 22,178 & 11,089 & 15 & 0.1 \\
\hline 2012 & 22,374 & 11,187 & 41 & 0.4 \\
\hline 2013 & 22,651 & 11,325 & 62 & 0.5 \\
\hline TOTAL & 281,620 & 140,808 & 1425 & 1.0 \\
\hline
\end{tabular}

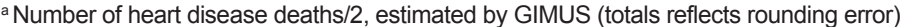
bICD-10 code 146.1[11]

GIMUS: Cuban Sudden Death Research Group SCD: sudden cardiac death

Source: Ministry of Public Health (CU) [12,18]

Is an SCD registry necessary? GIMUS has intensively researched SCD. An 11-year epidemiological series by Ochoa of over 10,000 natural deaths studied consecutively found that $10 \%$ were considered sudden.[10]

SD accounts for an estimated $50 \%$ of global cardiovascular mortality.[15-17] Based on this assumption, GIMUS estimated SCD occurrence in Cuba using data from the National Medical Records and Health Statistics Bureau (DNE) of the Ministry of Public Health (MINSAP) in 2013. Since 22,651 deaths from cardiovascular conditions were reported that year,[18] it could be assumed that some 11,325 of these deaths had been sudden, for a rate of $101 / 100,000$ population, or $12.3 \%$ of natural deaths that year-far more than the $0.04 \%$ reported for 2012.[12]

At the First Cuban Symposium on Sudden Cardiac Death, in 2013,[19] researchers working on SCD, and DNE representatives discussed the issue in depth. They agreed on the need to start building an official Cuban SCD registry. Applying ICD10 code 146.1 (Sudden cardiac death, so described)[11] to the 140,808 SDs GIMUS estimated for the period 2001-2013, SCD was recorded as cause of death on only $1 \%$ of death certificates (Table 1). The Symposium's conclusions included a proposal to discuss with DNE authorities the possibility of making sudden cardiac arrest notifiable,[19] for the purpose of demonstrating that emergency response systems should be organized to be faster and more efficient in cardiac emergencies.

On this topic, it is important to remember that heart disease was the leading cause of death in Cuba for over 40 years, until $2012,[20]$ when cancer overtook it by several hundred cases (cancer and heart disease mortality were 200.3 and 197.6 per 100,000 population, respectively). However, this was not due to a steady decline in heart disease deaths, but rather to an increase in rates of different types of cancer. It is considered advisable that, when an SCD occurs with an identifiable direct cause, SCD should be recorded on the death certificate together with the direct cause: e.g., Sudden death from acute myocardial infarction.

An official registry is needed for practical, not just academic, reasons. As long as the actual extent of the problem remains unknown, health authorities will be unable to formulate effective intervention strategies. A registry will enable development of strategies ranging from planning consistent health policies, led by MINSAP, to public education programs teaching basic first-responder measures and generally enhancing awareness of this problem. It should be noted that survival following an episode of sudden cardiac arrest has increased in countries where automated external defibrillators have been installed in public places; and police officers, firefighters, health workers, first responders, and even teenagers have been trained (through the public school system) in basic CPR and sudden cardiac arrest response.[4,7,19] Such strategies leading to greater public awareness and preparation could help prevent a substantial portion of SCDs (every 46 minutes, a Cuban dies of a heart attack). $[5,21]$

\section{Defining SCD and cutoff time for diagnosis}

Definition The definition of SD has been highly controversial, particularly since 1980 , when it ceased to be a purely epidemiological concept and became a defining element in prevention and effective treatment.[22] In fact, variability in the definition has resulted in inconsistencies, hindering comparisons among studies by different researchers, making it difficult to assess new therapies or carry out prospective followup to reduce high incidence.[15]

SD definition is based on one major inclusion criterion-its unexpected nature - and two minor inclusion criteria - its natural cause and time elapsed from symptom onset until death. SD may have a cardiovascular, neurological, respiratory or digestive cause, among others, depending on the affected system. The term cardiac is applied when the heart is the organ responsible for death. SCD accounts for $90 \%$ of SDs in general.[23]

Harrison's Principles of Internal Medicine defines SCD as natural death due to cardiac causes in a person who may or may not have previously recognized heart disease but in whom the time and mode of death are unexpected. In the context of time, 'sudden' is defined for most clinical and epidemiologic purposes as one hour or less between a change in clinical status heralding the onset of the terminal clinical event and the cardiac arrest itself. An exception is unwitnessed deaths, in which pathologists may expand the definition of time to $24 \mathrm{~h}$ after the victim was last seen to be alive and stable.[24]

Time elapsed Despite the gradual reduction in time elapsed in the definition, based on improved access to care in acute events, there is no consensus among different specialties on the use of one hour as the ideal cutoff time for SCD diagnosis. Pathologists extend the time up to six hours, which they consider essential for appearance of histological changes indicating myocardial necrosis due to ischemia,[23] a crucial point, considering that ischemia causes $80 \%$ of SCDs.[22]

Undoubtedly, lack of techniques available only in specialized facilities for identifying myocardial necrosis in less than six hours makes the one-hour time period untenable. We and other authors maintain that six hours from onset of deteriorated car- 
diac function is more appropriate.[25] This position is supported by Ochoa's finding that $85.7 \%$ of deaths (347 cases) from noninstantaneous SCD occurred between the first and sixth hour following symptom onset. The same study found that $60.8 \%$ of cases occurred in a prehospital setting, i.e., before reaching a facility where advanced cardiopulmonary cerebral resuscitation was available.[10]

Presence or absence of known heart disease There is a conflict between clinicopathological and forensic understanding concerning the unresolved issue of preexisting heart disease. This may well be the most important barrier to consensus on a definition, since it is contingent on the conceptual bases of both disciplines. On the forensic side, the indispensable condition is the absence of preexisting disease, which is conceptually the opposite of clinicopathological thinking.[26]

On the one hand, we clinicians consider it important to note that absence of preexisting heart disease makes it impossible to infer the immediate cause of an SD, because the definition of SCD specifies preexisting heart disease, whether or not known by the patient. On the other hand, we believe it inappropriate to exclude patients with known heart disease, as proposed by forensic scientists because, if a patient who has suffered a heart attack is now stable within the parameters for control of the disease, he or she is not expected to die suddenly. If such a person were to die after a sudden collapse of cardiovascular function, it would be a typical SCD from a clinicopathological standpoint. It would still be a natural, unexpected death from cardiovascular causes shortly after symptom onset. We need an in-depth discussion of the fundamentals of the definition, since unifying criteria is the starting point for an effective approach.

We propose the following definition of SCD for use in Cuba: an unexpected natural death from cardiovascular causes within six hours of onset of symptoms of deteriorated cardiac function in an individual with known or unknown preexisting heart disease.

If SCD is a natural death, why is it included in forensic medicine? What strategy should Cuba adopt? The field of forensic medicine is responsible for analyzing all deaths that are violent (self-evident definition from the adjective) or suspicious (in which there is any indication, medical or not, that a priori prevents ruling out a criminal cause).[23] SDs in general, and SCDs in particular, are prototypically suspicious deaths, not only because they are unexpected, but also because one third of all SCDs are unwitnessed.[15]

Forensic medicine is crucial to clarifying the nature of SD when it is impossible to deduce cause of death through logic or because of a lack of background information. Critical are the skill and thoroughness with which a forensic autopsy is performed, since this procedure addresses many more aspects than a clinical autopsy. Protocols that require a forensic autopsy when a death occurs under suspicious circumstances (or when the direct cause of death cannot be deduced) have yielded excellent results. Spain offers a case in point: a judicial order is issued, which triggers an entire system that enables prosecution when suspected criminal activity is confirmed (which logically implies that the case can no longer be considered SD). If, on the contrary, a natural cause of death is found, the death is recorded in the statistical information system as an SD (due to the particular cause).[23]
Cuba would benefit from adapting a similar approach to its own context. Logistic support would be needed from the Forensic Medicine Institute, which would depend on practical possibilities of the National Health System. For now, we advocate that all SDs where crime is ruled out should be subject to a clinical autopsy because most hospitals in Cuba have a pathology department. Such a protocol would eventually reduce over-reporting of acute myocardial infarction as an SCD cause (observed by the authors over our years of practice), and would bring us closer to the clinicopathological correlation so necessary for improving quality.

Pathological confirmation of cause of death is vital, as was demonstrated in Tavora's study of out-of-hospital SDs, finding only $50 \%$ accuracy in recorded direct cause of death on the death certificate, versus autopsy findings.[27] This highlights an important limitation of retrospective studies of SCD: lack of autopsy data in many cases.[4] Basso published guidelines for the European Union to ensure autopsy to confirm cause of death in SCD cases. [28] Clinical autopsy, then, is intrinsic to gaining a better understanding of the matter.

Who should specific intervention strategies target? Heart disease mortality rates in Cuba increased from 148.2 per 100,000 population in 1970[26] to 202.9 in 2013.[18] However, the precise incidence of SCD is unclear, but also indispensable for addressing this problem.

Tackling SCD requires stratification of risk. High-risk groups include: patients with known and demonstrated structural heart disease or conduction disorders and left ventricular ejection fraction $<35 \%$.[29] Although there is no denying that SCD mortality rates are higher in these groups, we sustain that concentrating only on them can miss important social dimensions. Mortality rates are highest in such groups-and somewhat less predictablethan in intermediate-risk groups (patients with acquired structural, hypertensive, valvular or coronary heart disease, provided that elements of acute myocardial ischemia or potentially fatal arrhythmias have been ruled out) and lower-risk groups (the general population with established cardiovascular risk factors-hypertension, diabetes, sedentary lifestyle, smoking, dyslipidemia). However, since the latter two groups are much larger, absolute number of events (heart attacks, deaths and SCDs) is greater, even though rates are lower (Figure 1).[29] This problem is known as the prevention paradox.[30]

Intervention strategies for high-risk groups range from drug therapy to implantable automatic defibrillators. In our view, strategies for patients in intermediate- or lower-risk groups, where $90 \%$ of events occur, should be aimed at controlling risk factors and chronic non-communicable diseases to prevent ischemic heart disease, which is responsible for as much as $80 \%$ of reported SCDs[15] and is on the rise in Cuba.[18] Prevention measures must be accompanied by professional and technological support that enables prompt and effective responses to calls for assistance in cases of out-of-hospital sudden cardiac arrest.

The First Cuban Symposium on SCD proposed sharing information with MINSAP on concrete strategies that in other settings have demonstrated an impact on lower-risk groups. These include training in basic and advanced CPR, according to level and profession; inclusion in all levels of the education system of a CPR class as a prerequisite to obtaining a driver's license; availability of auto- 
Figure 1: SCD distribution by population risk level, percentage and absolute numbers

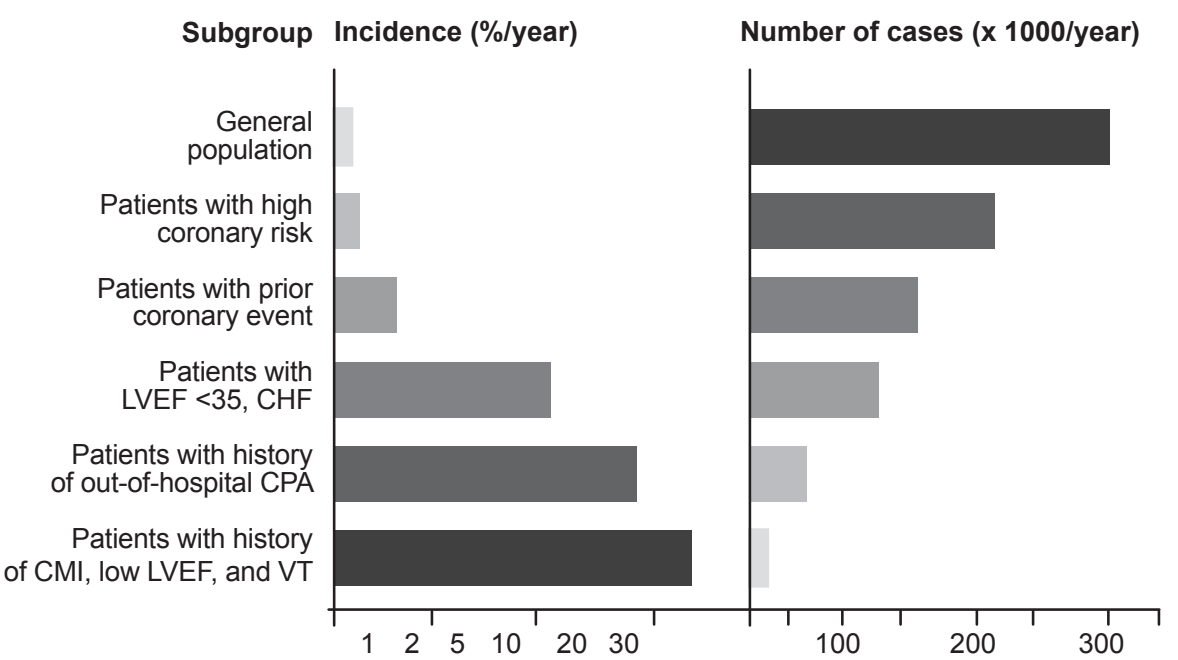

CHF: congestive heart failure CMI: chronic myocardial infarction LVEF: left ventricular ejection fraction VT: ventricular tachycardia CPA: cardiopulmonary arrest Source: Myerburg[24]

mated defibrillators in highly frequented places; and changes in the approach to systematic physical exercise (i.e., promoting orderly regimens modified according individual characteristics and health status), among other strategies to reduce not only SCD incidence, but overall cardiovascular mortality as well.[19]

In conclusion, we consider that intervention strategies must be balanced. Without ignoring high-risk patients or dismissing the impact of scientific advances on reducing mortality among them, we need to develop concrete policies focused on intermediateand lower-risk groups, such as those described above.

Is SCD "just another way to die"? If someone suffers a heart attack and progresses to SCD, there simply are no therapeutic options, such as post-stroke rehabilitation or dialysis for chronic renal failure. SCD's impact lies in its abrupt and unexpected nature, without treatment options to change the outcome. This is reason enough to consider SCD not just another way to die but, rather, a process that requires special care and that can and should be prevented.
The natural history of SCD involves four stages: prodrome, onset of terminal event, cardiac arrest, and biological death. Cardiac arrest is potentially reversible by appropriate and timely interventions.[24] Sudden cardiac arrest is a sudden interruption of the heart's pumping function that is reversible with immediate intervention but will end in death if such an intervention is not undertaken. [24] International experts believe that sudden cardiac arrest should be included as a notifiable disease because of its magnitude, importance and costs for health systems worldwide. $[5,31,32]$ Such mandatory reporting could contribute to a clearer statistical picture of the extent of the SCD problem in Cuba.

The First Cuban Symposium on Sudden Cardiac Death, attended by delegates from 12 countries and 13 Cuban health sciences societies,[33] was an important first step for the Cuban scientific community's in-depth study of the topic, examining its importance as a global and Cuban health problem. Guidelines emerged for immediate steps to be taken by specialists involved in SCD in a multi- and interdisciplinary approach.

\section{CONCLUSIONS}

As conclusions, we offer the GIMUS position on SCD:

- It is vital to develop and implement an official Cuban SCD registry.

- The generally accepted Cuban clinical definition of SCD meets scientific requirements, but the cutoff time should be six hours from onset of deteriorated cardiac function to certification of death.

- Cases with known heart disease should not be excluded from the SCD definition.

- A clinical autopsy should be required in all SCD cases cleared of criminal suspicion.

- Health policies should target all groups at risk for SCD, focusing on the lower-risk general population, with concrete actions (with measureable medium- and long-term impacts) aimed at its reduction. -1 -

\section{REFERENCES}

1. Holmberg M, Holmberg S, Herlitz J. The problem of out-of-hospital cardiac arrest prevalence of sudden death in Europe today. Am J Cardiol. 1999;83:88-90.

2. Brugada P. Epidemiology of sudden cardiac death. Rev Esp Cardiol Supl. 2013;13(Suppl A):2-6.

3. Morentin B, Audicana C. Estudio poblacional de la muerte súbita cardiovascular extrahospitalaria: incidencia y causas de muerte en adultos de edad mediana. Rev Esp Cardiol. 2011;64(1):2834. Spanish.

4. Vigo-Ramos J. Muerte súbita y emergencias cardiovasculares: problemática actual. Rev Peru Med Exp Salud Pública. 2008;25(2):233-6. Spanish.

5. Curós Abadal A. Parada cardíaca extra hospitalaria, nuestra asignatura pendiente. Rev Esp Cardiol. 2001;54(7):827-30. Spanish.

6. Kuller LH. Sudden death. Definition and epidemiologic considerations. Prog Cardiovasc Dis. 1980 Jul-Aug;23(1):1-12

7. Gold LS, Fahernbruch CE, Rea TD, Eisenberg MS. The relationship between time to arrival of emergency medical services and survival from out-of- hospital ventricular fibrillation cardiac arrest. Resuscitation. 2010 May;81(5):622-5.

8. Valenzuela TD, Roe DJ, Nichol G, Clark LL, Spaite DW, Hardman RG, et al. Outcomes of rapid defibrillation by security officers after cardiac arrest in casinos. N Engl J Med. 2000;343(17):1206-9.

9. García Montero A, García Vega ME, Quiñones Zamora A, Chávez Pérez E, Cañedo Hernández O. Impacto del sistema integral de urgencias médicas en la mortalidad por infarto miocárdico agudo. Rev Cub Med Int Emerg. 2006;5(3):3126. Spanish.

10. Ochoa Montes LA. Muerte súbita cardíaca. Estudio en comunidades de Arroyo Naranjo en el período 2000-2010 [thesis]. [Havana]: Atherosclerosis Research and Reference Center; 2012 [cited 2014 Jul 2]. Available from: http://tesis .repo.sld.cu/639/. Spanish.

11. World Health Organization; Pan American Health Organization. Clasificación Estadística Interna- cional de Enfermedades y Problemas relacionados con la Salud 10th ed [Internet]. Washington, D.C.: World Health Organization; 2008 [cited 2014 Jul 2]. Available from: http://www.sld.cu/ sitios/dne/temas.php?idv=4007. Spanish.

12. National Health Statistics and Medical Records Division (CU). Reporte estadístico de la muerte súbita cardiovascular (2001-2013). Havana: Ministry of Public Health (CU); 2013. Spanish.

13. Ochoa Montes LA. Un error sobre otro error. Revista Cubana Salud Pública. 2013;39(2):420-2.

14. Interamerican Society of Cardiology. [Internet] Mexico, D.F.: Interamerican Society of Cardiology; c2015. Consejos. Alianza Contra la Muerte Súbita; [cited 2015 Sep 15]; [about 3 screens] Available from: http://www.siacardio.com/ca tegory/consejos/alianza-contra-la-muerte-subi ta/. Spanish.

15. Chugh SS, Reinier K, Teodorescu C, Evanado A, Kehr E, Al Samara M, et al. Epidemiology of sudden cardiac death: clinical and research 
implications. Prog Cardiovasc Dis. 2008 NovDec;51(3):213-28.

16. Garillo R. Muerte súbita cardiaca: las siete preguntas para las cuales todo cardiólogo debe tener respuesta. Arch Cardiol Mex. 2010 JulSep;80(3):199-204. Spanish.

17. Muratore C, Belziti C, Gant López J, Di Toro D, Mulassi A, Corte M, et al. Incidencia y variables asociadas con la muerte súbita en una población general. Sub-análisis del estudio PRISMA. Rev Argent Cardiol. 2006 Nov-Dec;74(6):441-6. Spanish.

18. Ministry of Public Health (CU). Serie de Informes Técnicos de la Dirección Nacional de Estadísticas y Registros Médicos. Anuario Estadístico de Salud. Havana: Ministry of Public Health (CU); 2013. p. 33. Spanish.

19. Informe del I Simposio Cubano de Muerte Súbita Cardiovascular. CorSalud [Internet]. 2014 [cited 2014 Jul 2];6(Suppl 1):101-4. Available from: http://www.cardiovcl.sld.cu/corsalud/2014/ v6s1a14/CorSalud6\%28Supl1\%292014.pdf. Spanish.

20. Ministry of Public Health (CU). Serie de Informes Técnicos de la Dirección Nacional de Estadísticas y Registros Médicos. Anuario Estadístico de Salud. Havana: Ministry of Public Health (CU); 2012. p. 31. Spanish.

21. Abella B, Aufderheide TP, Eigel B, Hickey RW, Longstreth WT Jr, Nadkarni V, et al. Reducing barriers for implementation of Bystander-Initiated Cardiopulmonary Resuscitation: a scientific statement from the American Heart Association for healthcare providers, policymakers, and community leaders regarding the effectiveness of cardiopulmonary resuscitation. Circulation. 2008 Feb 5;117(5):704-9.

22. Bayés de Luna A, Elousa R. Muerte súbita. Rev Esp Cardiol. 2012;65(11):1039-52. Spanish.
23. Castellá García J, Medallo Muñiz J, Marrón Moya T. Aspectos medicolegales de la muerte súbita cardiaca. Rev Esp Cardiol Supl. 2013;13(Suppl A):30-7. Spanish.

24. Myerburg RL, Castellanos A. Arrest, and Sudden Cardiac Death. In: Longo DL, Fauci AS, Kasper DL, Hauser SL, Jameson JL, Loscalzo J, editors. Harrison's Principles of Internal Medicine. 18th ed. Chapter 273. New York: Mc Graw-Hill Medical; 2013.

25. Virmani R. Sudden cardiac death In: Virmani $R$, Atkinson JB, Burke A, Farb A, editors. Cardiovascular Pathology. 2nd ed. Philadelphia: WB Saunders; 2001 Jun 29. p. 340-85.

26. Pérez Álvarez H, Ferrer Marrero D. Aspectos médico-legales de la muerte súbita cardiovascular. CorSalud. 2014;6(Suppl 1):65-70. Spanish.

27. Tavora F, Crowder C, Kutys R, Burke A. Discrepancies in initial death certificate diagnoses in sudden unexpected out-of-hospital deaths: the role of cardiovascular autopsy. Cardiovasc Pathol. 2008 May-Jun;17(3):178-82.

28. Basso C, Burke M, Fornes P, Gallagher PJ, de Gouveia RH, Sheppard M, et al. Guidelines for autopsy investigation of sudden cardiac death. Virchows Arch. 2008 Jan;452(1):11-8.

29. Myerburg RJ, Kessler K, Castellanos A. Sudden cardiac death: structure, functions and timedependence of risk. Circulation. 1992 Jan;85(1 Suppl):I2-10.

30. Rose G. Sick individuals and sick populations. Int J Epidemiol. 1985 Mar;14(1):32-8.

31. López Messa JB. ¿Debe el paro cardiaco extrahospitalario ser una enfermedad de declaración obligatoria? Rev Electrónica Med Intensiva. 2008 May;8(5). Spanish.

32. Berdowski J, Berg RA, Tijssen JGP, Koster RW. Global incidences of out-of hospital cardiac arrest and survival rates: systematic review of
67 prospective studies. Resuscitation. 2010 Nov;81(11):1479-87.

33. I Simposio Cubano de Muerte Súbita Cardiovascular. CorSalud. 2014;6(Suppl 1):14-6. Spanish

\section{THE AUTHORS}

Ernesto Vilches Izquierdo (Corresponding author: evilches84@gmail.com), internist with master's degree in atherosclerosis research. Vice president, Sudden Death Research Group (GIMUS), Clinical director, Cuando Cubango Medical Clinic and Pharmacy Clinic, Menongue, Angola.

Luis A. Ochoa Montes, dual specialist in internal medicine and cardiology with doctorate in medical sciences. Full professor and senior researcher, Hermanos Ameijeiras ClinicalSurgical Teaching Hospital; president, GIMUS, Havana, Cuba.

Lianne Ramos Marrero, dual specialist in family and internal medicine. Instructor, René Bedia Morales Teaching Polyclinic, GIMUS member, Havana, Cuba.

Submitted: January 8, 2014

Approved for publication: October 1, 2015

Disclosures: None

\title{
MEDICC Review Themes for Upcoming Issues
}

\author{
2016-2017 : Intersectoral Initiatives for Population Health \\ Challenges of Healthy Aging \\ Communication, Culture \& Health \\ The Big Risks: Cancer \& Vascular Diseases
}

Vol. 17, No. 2, 2015

Environment, Climate Change \& Health

Vol. 17, No. 1, 2015

Maternal \& Child Health

Vol. 16, No. 3-4, 2014

Dual Themes: Cancer \& Genetics 